MODELING, IDENTIFICATION AND CONTROL, 2004, VOL. 25, No. 3, 173-190

doi:10.4173/mic.2004.3.3

\title{
A Toolbox of Aiding Techniques for the HUGIN AUV Integrated Inertial Navigation System*
}

\author{
BJORN JALVING $\dagger$, KENNETH GADE $\dagger$, OVE KENT HAGEN $\dagger$ and \\ KARSTEIN VESTGÅRD
}

Keywords: Autonomous underwater vehicle, aided inertial navigation, Kalman
filter, Doppler velocity log, synthetic aperture sonar, GPS. USBL, transponder
navigation, terrain navigation smoothing

Modern AUV designs must handle submerged autonomous operation for long periods of time. The state of the art solution embedded in the HUGIN AUVs is a Doppler Velocity Log (DVL) aided Inertial Navigation System (INS) that can integrate various forms of position measurement updates. In autonomous operations, position updates are only available in limited periods of time or space, thus the core velocity aided inertial navigation system must exhibit high accuracy. However, position uncertainty of a DVL aided inertial navigation system will eventually drift off, compromising either mission operation or requirements for accurate positioning of payload data. To meet the requirements for a range of military and civilian AUV applications, the HUGIN vehicles come with a flexible and powerful set of navigation techniques. Methods for position updates include GPS surface fix, DGPS-USBL, Underwater Transponder Positioning (UTP) and bathymetric terrain navigation. Based on synthetic aperture sonar technology, a potentially revolutionary accurate velocity measurement is under development. HUGIN also comes with a navigation post-processing system (NavLab), which can be applied to increase navigational integrity and maximize position accuracy.

\section{Introduction}

Autonomous Underwater Vehicles (AUVs) have in recent years convincingly demonstrated their capabilities in real applications. Civilian applications include detailed seabed mapping, environmental monitoring and research and inspection work for offshore industry. Short time frame military applications include Mine Counter Measures (MCM) and Rapid Environmental Assessment (REA). In a longer time frame, AUVs will play an important part in the general robotization of modern warfare.

Kongsberg Maritime and FFI have cooperated in developing the HUGIN family of autonomous underwater vehicles. HUGIN 3000 was the world's first AUV used in commercial survey operations (Chance et al., 2000 and Vestgård et al., 2001). The four HUGIN AUVs currently in service have been used in areas as diverse as the Gulf of Mexico, the Mediterranean, Brazil, West Africa, the North Sea and the Norwegian Sea. Building on more than 5 years of field experience with commercial AUV use, the HUGIN 1000 vehicle is now under development (first delivery end 2003), targeting the military market and civilian environmental monitoring and

*(C)2003 MTS and IEEE. Reprinted with permission from Proceedings from Oceans 2003, September 23-25, San Diego, CA, USA.

†Norwegian Defence Research Establishment (FFI), P.O. Box 25, 2027 Kjeller, Norway, emails: Bjorn.Jalving@ffi.no, Kenneth.Gade@ffi.no, Ove-Kent.Hagen@ffi.no

\$Kongsberg Maritime, P.O. Box 111, 3191 Horten, Norway, email: Karstein.Vestgard@, kongsbergmaritime.com 
research. Compared to HUGIN 3000, HUGIN 1000 is smaller, easier to handle, has lower depth rating and shorter endurance, but software, electronics and system design are almost identical (Hagen et al., 2003b). This paper discusses the design of the integrated inertial navigation system for the HUGIN family and the development of a toolbox of navigation techniques to meet the requirements for a range of AUV applications.

\section{HUGIN Integrated Inertial Naviation System}

\subsection{Integrated Inertial Navigation System Structure}

In Figure 1, the structure of the HUGIN integrated inertial navigation system is shown. The Inertial Navigation System (INS) calculates position, velocity and attitude

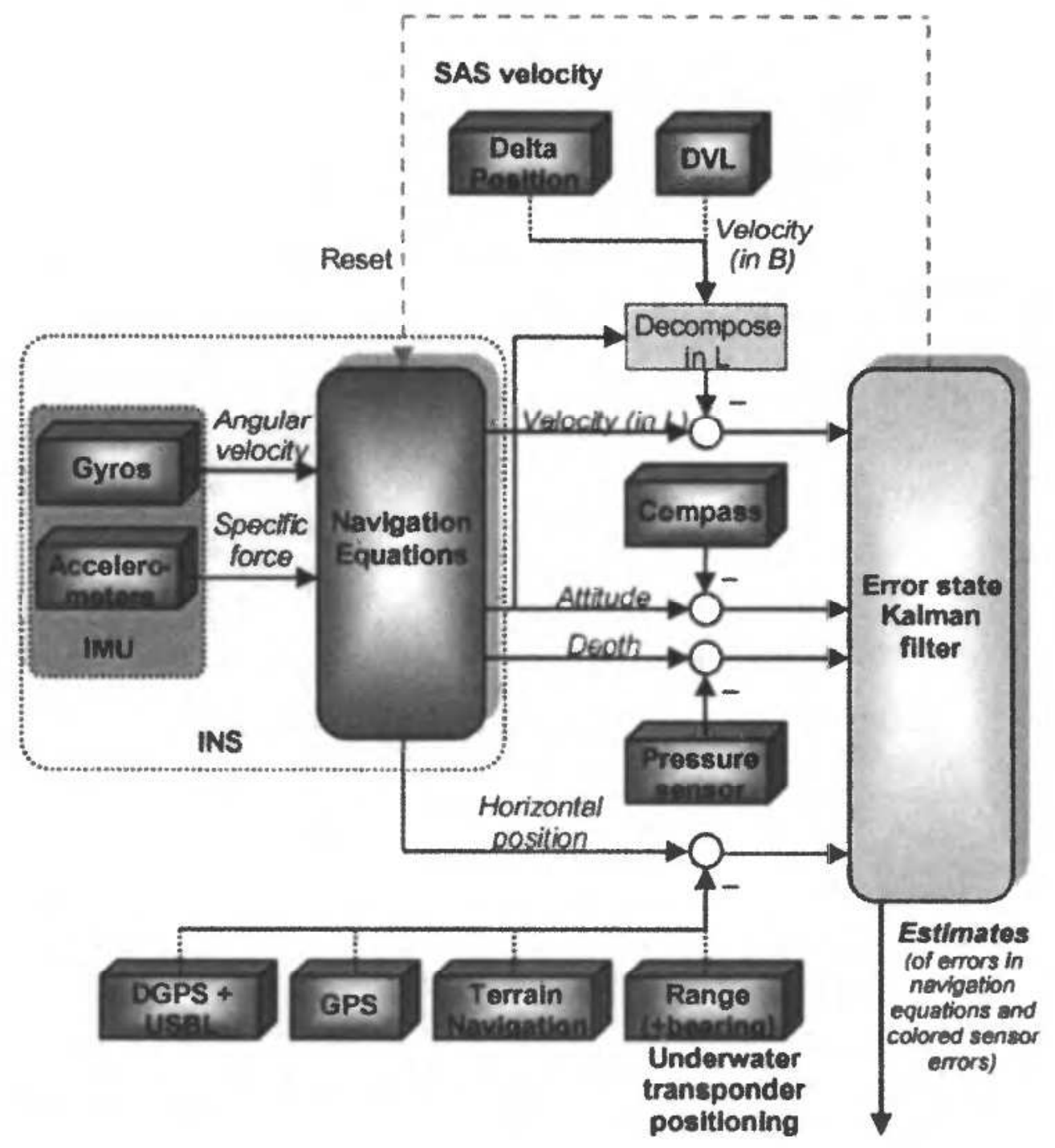

Figure 1. HUGIN integrated inertial navigation system structure. 
using high frequency data from an Inertial Measurement Unit (IMU). An IMU consists of three accelerometers measuring specific force and three gyros measuring angular rate. A Kalman filter will, in a mathematically optimal manner, utilize a wide variety of navigation sensors for aiding the INS. The Kalman filter is based on an error-state model and provides a much higher total navigation performance than is obtained from the independent navigation sensors.

\subsection{DVL aided INS - Core Navigation System}

2.2.1. DVL Aided INS Autonomous operation in deep water or covert military operations requires the AUV to handle submerged operation for long periods of time. The solution for modern AUVs is a low drift Doppler Velocity Log (DVL) aided inertial navigation system that can integrate various forms of position measurement updates. In Figure 1, the core DVL aided INS system consists of the IMU and the navigation equations, the error state Kalman filter and DVL, compass (optional) and pressure aiding sensors.

Inertial navigation systems are usually classified by the standard deviation of the positional error growth of their free inertial (unaided) performance (see Table 1). A free inertial INS will, after a short period of time, have unacceptable position errors. The HUGIN navigation system can in principle interface any IMU, but for most applications the IMU will be in the $1 \mathrm{nmi} / \mathrm{h}$ class.

DVL accuracy is dependent on frequency. Higher frequency yields better accuracy at the sacrifice of decreased range as illustrated in Table 2. Prioritization between range and accuracy is dependent on the application.

2.2.2. Simplified Error Analysis Straight Trajectories The simplified error analysis presented in this section is useful for understanding the basic mechanisms of a VVL aided INS and assessing how IMU and DVL sensor accuracy is determining the overall position accuracy.

The horizontal position drift in a DVL-aided INS is determined by the error in the estimated Earth-fixed velocity (i.e. North and East velocity). The main contributors to this error are:

Table 1. INS classes. Notes 1: RLG-Ring Laser Gyro, FOG-Fiber Optic Gyro

\begin{tabular}{lccc}
\hline Class & Gyro technology & Gyro bias & Accelerometer bias \\
\hline$>10 \mathrm{nmi} / \mathrm{h}$ & RLG, FOG & $1 \% \mathrm{~h}$ & 1 milli g \\
$1 \mathrm{nmi} / \mathrm{h}$ & RLG, FOG & $0.005^{\circ} / \mathrm{h}$ & 30 micro $\mathrm{g}$ \\
\hline
\end{tabular}

Table 2. RDI Workhorse Navigator Doppler Velocity Log accuracy and range specifications (RD Instruments, 2003). o.s. - of speed.

\begin{tabular}{ccc}
\hline Frequency & Long term accuracy & Range \\
\hline $150 \mathrm{kHz}$ & $\pm 0.5 \%$ o.s. $\pm 2 \mathrm{~mm} / \mathrm{s}$ & $425-500 \mathrm{~m}$ \\
$300 \mathrm{kHz}$ & $\pm 0.4 \%$ o.s. $\pm 2 \mathrm{~mm} / \mathrm{s}$ & $200 \mathrm{~m}$ \\
$600 \mathrm{kHz}$ & $\pm 0.2 \%$ o.s. $\pm 1 \mathrm{~mm} / \mathrm{s}$ & $90 \mathrm{~m}$ \\
$1200 \mathrm{kHz}$ & $\pm 0.2 \%$ o.s. $\pm 1 \mathrm{~mm} / \mathrm{s}$ & $30 \mathrm{~m}$ \\
\hline
\end{tabular}


- Error in the body-fixed velocity

- Error in heading.

The error in estimated body fixed velocity, is mainly determined by the lowfrequency error in the DVL itself (without position aiding this error is not observable when going at a straight line). High frequency velocity errors are estimated by means of the accelerometers. Even the most accurate INS will without aiding, after a short period of time have a velocity uncertainty larger than the DVL accuracy. Referring to Table 2, a $300 \mathrm{kHz}$ DVL typically have a scale factor type of error of $0.4 \%$ of speed, contributing to an along track error drift of $0.4 \%$ of traveled distance, or 28.8 $\mathrm{m} /$ hour for an AUV traveling at $2 \mathrm{~m} / \mathrm{s}$ ( 4 knots). However, there are ways to improve the DVL accuracy. Sacrificing range, the $1200 \mathrm{kHz}$ version from the same vendor has an accuracy specification of $0.2 \%$ of speed, corresponding to $0.2 \%$ of traveled distance, or $14.4 \mathrm{~m} / \mathrm{hour}$ (AUV speed $2 \mathrm{~m} / \mathrm{s}$ ). The scale factor error is observable by the Kalman filter when position measurements are available or when the AUV is turning. Thus, the Kalman filter can compensate for part of the scale factor error when running more complex missions than a straight line. This is discussed in Section 2.2.3.

The error in heading is determined by the gyrocompassing capability of the integrated system. The heading estimation error will typically be of low frequency, corresponding to non-observable gyro bias dynamics. Referring to Table 1, a $1 \mathrm{nmi} /$ $\mathrm{h}$ navigation class IMU typically gyrocompass to an accuracy of $\sigma(\delta \psi)=0.02 \mathrm{deg}$ sec latitude. This corresponds to an error drift of $\sigma(\delta \psi) \cdot 100 \%$ of traveled distance $\left(\sigma(\delta \psi)\right.$ in radians). At $45^{\circ}$ latitude, this equals $0.05 \%$ of traveled distance, or $3.4 \mathrm{~m} /$ hour at $2 \mathrm{~m} / \mathrm{s}$ AUV speed.

In Jalving et al. (2003a) position accuracy for an INS with $1 \mathrm{nmi} / \mathrm{h} \mathrm{IMU} \mathrm{and}$ $1200 \mathrm{kHz}$ DVL following a straight line was simulated. Along track position error drift was in the order of $8 \mathrm{~m} /$ hour while cross track position error drift was in the order of $2.5 \mathrm{~m} /$ hour. This is a somewhat smaller drift than predicted by the simplified error analysis. There are two main reasons; the Kalman filter compensates for a scale factor error estimated when position measurements were available and the actual scale factor error is modeled as a first order Markov process and not a constant error. Choosing time constants that realistically reflect the physical error process is very important when estimating DVL aided INS error drift and when tuning the Kalman filter for real applications.

Since $1 \mathrm{nmi} / \mathrm{h}$ navigation class IMUs are relatively easily obtainable in the marketplace and the DVL induced position error is close to an order of magnitude larger than the IMU induced position error for straight line trajectories, most focus should actually be on how to improve the velocity accuracy. This explains the importance of the work presented in Section 2.3.

2.2.3. Countering DVL Aided INS Position Error Growth For a submerged AUV without position updates, the position error growth of a DVL aided INS can be countered by:

1. Mission pattern for canceling of error growth

2. Kalman filter estimation and compensation of DVL error

The accuracy estimates in Section 2.2.2 are valid for straight-line trajectories. Since the main error contributors of DVL aided INS is body fixed velocity and heading, a canceling effect of the error growth is obtained when for instance running 
Table 3. Typical reduction in position error drift for a DVL aided INS when comparing a straight-line trajectory with a lawn mower pattern (Jalving et al., 2003a). The numbers apply for a $1200 \mathrm{kHz}$ DVL and a $1 \mathrm{nmi} / \mathrm{h} \mathrm{IMU}$ at $45^{\circ}$ latitude.

\begin{tabular}{lcc}
\hline $\begin{array}{l}\text { Position error drift } \\
\text { (\% of traveled distance) }\end{array}$ & Straight line & $\begin{array}{c}\text { Lawn mower pattern } \\
\text { with 1 km lines }\end{array}$ \\
\hline Along track & $0.11 \%$ & $0.01 \%$ \\
Across track & $0.03 \%$ & $0.001 \%$ \\
\hline
\end{tabular}

a lawn mower pattern. The canceling effect increases with the stability of the body fixed velocity error and heading error. Also the canceling effect increases with shorter line lengths.

A second important effect of maneuvering is that the velocity error actually becomes observable by means of comparing expected centripetal acceleration with measured acceleration from the IMU. If the velocity error is the same during the maneuver (i.e. when it is observed) as it is in the following line, this estimation will significantly reduce the drift. However real DVL-data from RDI Workhorse Navigator $300 \mathrm{kHz}$ shows that during the maneuver the error might be different, and in such cases this effect will have limited importance for the overall position drift. This real data problem can be countered by a sophisticated compensation method, but preferably, other sensors or frequencies might not exhibit this error characteristic. When the mechanism works, the error growth when running long straight lines can be significantly reduced by adding $360^{\circ}$ turns at regular intervals.

The two effects combined are very effective, as seen in Table 3, which contains results from NavLab simulations (see Section 2.9 for NavL ab description).

\subsection{SAS Velocity Aiding}

In Section 2.2.2 it was shown that for an AUV equipped with a $1 \mathrm{nmi} / \mathrm{h}$ type of IMU or better, the DVL accuracy is the limiting factor to the position accuracy during submerged navigation with no position updates.

Modern MCM and REA AUVs are likely to be equipped with Synthetic Aperture Sonar (SAS) due to the improved resolution and image quality offered compared to Side Scan Sonar (SSS). SAS requires very good relative navigation to obtain focused images. Relative navigation in SAS over a synthetic aperture is often referred to as micro-navigation. One method of micro-navigation, called Displaced Phase Center Antenna (DPCA), generates, as a by-product, a revolutionary good velocity (or more precisely, displacement) measurement. This complex displacement measurement needs to be integrated in the Kalman filter in a non-traditional way, which is an on-going research effort.

The DPCA velocity measurement technique, based on expensive and sophisticated sonar hardware and advanced signal processing, is in fact very similar to the technique used in a Correlation Velocity Log (CVL). If expectations are proved true and the DPCA velocity measurement is an order of magnitude more accurate than DVL, along track error contribution will be in the same order as across track error contribution. Consequently a leap in performance of velocity aided inertial navigation systems has been achieved, allowing longer time intervals between position updates. 


\subsection{GPS Surface Fix}

As seen in Figure 1, there are several alternatives for providing the integrated inertial navigation systems with position updates. GPS surface fix is the most intuitive method and should be applied when possible. The following GPS services can be used:

- GPS Standard Positioning Service (SPS)

- GPS Precise Positioning Service (PPS)

- Differential GPS (DGPS)

- Real-Time Kinematic GPS (RTKGPS)

GPS SPS is available to all users worldwide. GPS PPS is available only to authorized users and primarily intended for military purposes. GPS PPS receivers should be the choice for military AUVs, at least for operations in denied areas. Compared to GPS SPS, GPS PPS is more resistant to jamming and deception. GPS SPS and GPS PPS have comparable accuracy. AUVs for detailed seabed mapping will typically be equipped with DGPS, or in some cases even RTKGPS.

\subsection{Combined DGPS-USBL (Ultra Short Base Line)}

In deep water seabed mapping, deploying and following the AUV with a survey vessel is the preferred method for obtaining maximum position accuracy. The survey vessel is equipped with differential GPS and tracks the AUV with an USBL system. Combined DGPS-USBL position measurements are transmitted to the AUV at regular intervals to bind the position error drift. See Section 4.1 for operational results.

\subsection{LBL (Long Base Line)}

LBL systems provide accurate AUV position measurements once a network of four LBL transponders has been deployed and calibrated. In principle, the HUGIN inertial navigation system can easily be integrated with a LBL system. However, the operational efforts involved in deployment and calibration is drastically reduced with underwater transponder positioning (Section 2.7), where only one underwater transponder is necessary to bind the INS position drift. LBL systems in AUV applications will probably become obsolete with the advent of this new navigation technique.

\subsection{Underwater Transponder Positioning (UTP)}

2.7.1. Old Principle - Revolutionary Solution Pinging a transponder on the seafloor and measuring range and bearing is the traditional approach to acoustic navigation. From range and bearing measurements, position has been computed in commercial Ultra Short Base Line (USBL) and Short Base Line (SBL) systems for decades. Instead of integrating a complex USBL system in an AUV, the AUV can be fitted with two transducers separated by as long baseline as possible (this is basically a SBL system).

This principle is called Underwater Transponder Positioning (UTP) and is the result of a joint development effort by FFI and Kongsberg Simrad. Kongsberg Simrad has delivered UTP to the American survey company C\&C Technologies on a commercial basis. The range and bearing measurements are tightly integrated as 
position measurements in the Kalman filter of the inertial navigation system (actually position measurements can be produced with only range measurements available as well). The system works with only one underwater transponder, but can utilize any number of transponders in an optimal way. Compared to a traditional LBL system, UTP has improved accuracy due to tight coupling with the INS, increased operating area and significantly less deployment costs, since only one transponder is necessary to bind the position drift.

2.7.2. Concurrent Deployment and Navigation (CDN) Current version of UTP requires that a survey vessel equipped with USBL box in the position of the underwater transponders. The transponder position coordinates must be sent to HUGIN prior to UTP navigation. In the next version, the HUGIN navigation system will be able to estimate the position of an underwater transponder while navigating with another. In this way, the AUV will be able to deploy a trail of underwater acoustic buoys for UTP navigation and acoustic communication. This concept can be denoted Concurrent Deployment and Navigation (CDN) or UTP CDN.

\subsection{Bathymetric Terrain Navigation}

2.8.1. Correlation Methods Terrain correlation may be done for one measurement, or on a sequence of measurements. The measured water depths are shifted around an offset area around current position estimate, and a correlation between the measurements and the depth data in the Digital Terrain Model (DTM) is calculated in this area. The calculated correlation is called the correlation surface. The correlation surface is analysed to determine convergence, calculating a position offset, the error covariance and a position fix confidence.

Terrain correlation runs on any sensor providing bathymetric data, for instance multibeam echosounder (MBE), altimeter, DVL or interferometric sonar. Terrain navigation accuracy depends on sensor accuracy, map accuracy, map resolution and not least terrain suitability.

In Figure 2 the HUGIN terrain correlation system is illustrated. The Geographic data producer converts AUV depth + bathymetric sensor data in AUV body-fixed coordinates to geographical referenced data, using the current navigation solution. The Terrain Correlator runs the terrain correlation algorithm on one measurement or iteratively on a sequence of measurements. Map Database readies the DTMs for random access by the Terrain Correlator. Position updates are sent to the integrated inertial navigation system Kalman filter to bind the INS position drift.

The actual correlation can be done selecting different algorithms:

- Terrain Contour Matching (TERCOM) (Golden, 1980)

A well-proven and robust algorithm that uses the mean absolute distance as a correlation measurement. Models for sensor and map noise may be included. The covariance matrix of the position fix is found through the correlation surface.

- Point Mass Filter (PMF) (Bergman et al., 1999)

A more sophisticated algorithm that actually calculates the position Probability Density Function (PDF) using Bayesian estimation. PMF enables the use of advanced models for sensor and map noise and enables a statistically sound use of the navigation system accuracy as an input. The covariance matrix of the position fix is found directly from the PDF. 


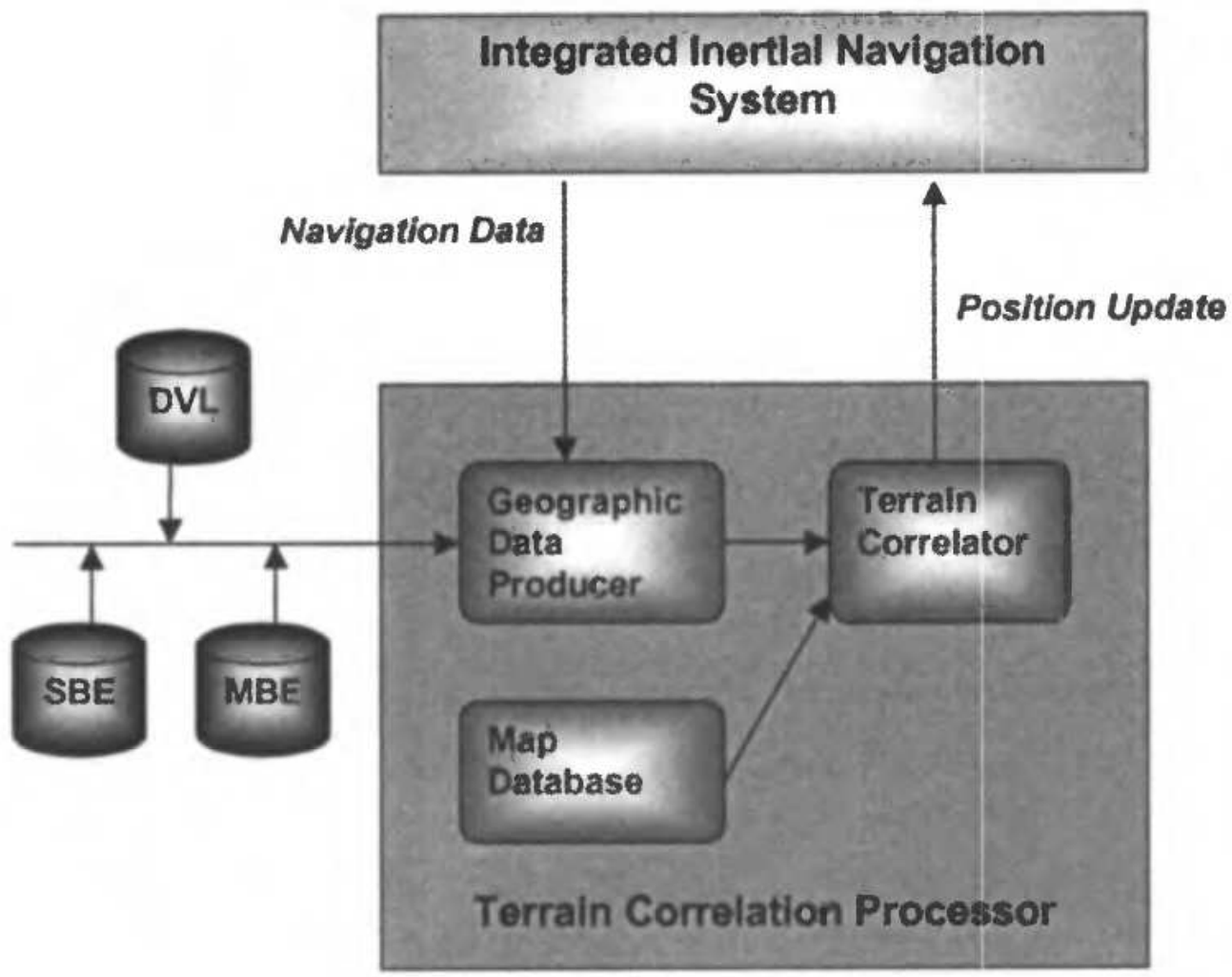

Figure 2. Structure of the HUGIN terrain correlation system.

2.8.2. Tightly Integrated Terrain-Tracking Algorithms Terrain navigation algorithms can conceptually be divided into correlation based global search algorithms (described in Section 2.8.1)) and tightly integrated terrain tracking algorithms. The latter are characterized by integration of range measurements and the bathymetric map into the Kalman filter. Thus, all available information in the integrated navigation system is utilized. Compared to correlation methods, the algorithms have less robust behavior in highly non-linear terrain. FFI has invested a considerable effort in developing a terrain-tracking algorithm called TRIN (Hagen \& Hagen, 2000). This is planned for integration in HUGIN, following the completion of the work on correlation-based methods.

2.8.3. Concurrent Mapping and Navigation $(C M N)$ An attractive feature of tightly integrated terrain-tracking algorithms is that a solution for Concurrent Mapping and Navigation (CMN) follows inherently.

Similar to UTP CDN, CMN is important to missions in unknown or denied areas. Solutions to CMN, considering both tightly integrated terrain-tracking algorithms and correlation algorithms, is an ongoing research effort.

\subsection{NavLab (Navigation Laboratory)}

NavLah (Navigation Laboratory), Gade (2003) and Gade (2004/5), is a powerful and versatile tool intended for: 


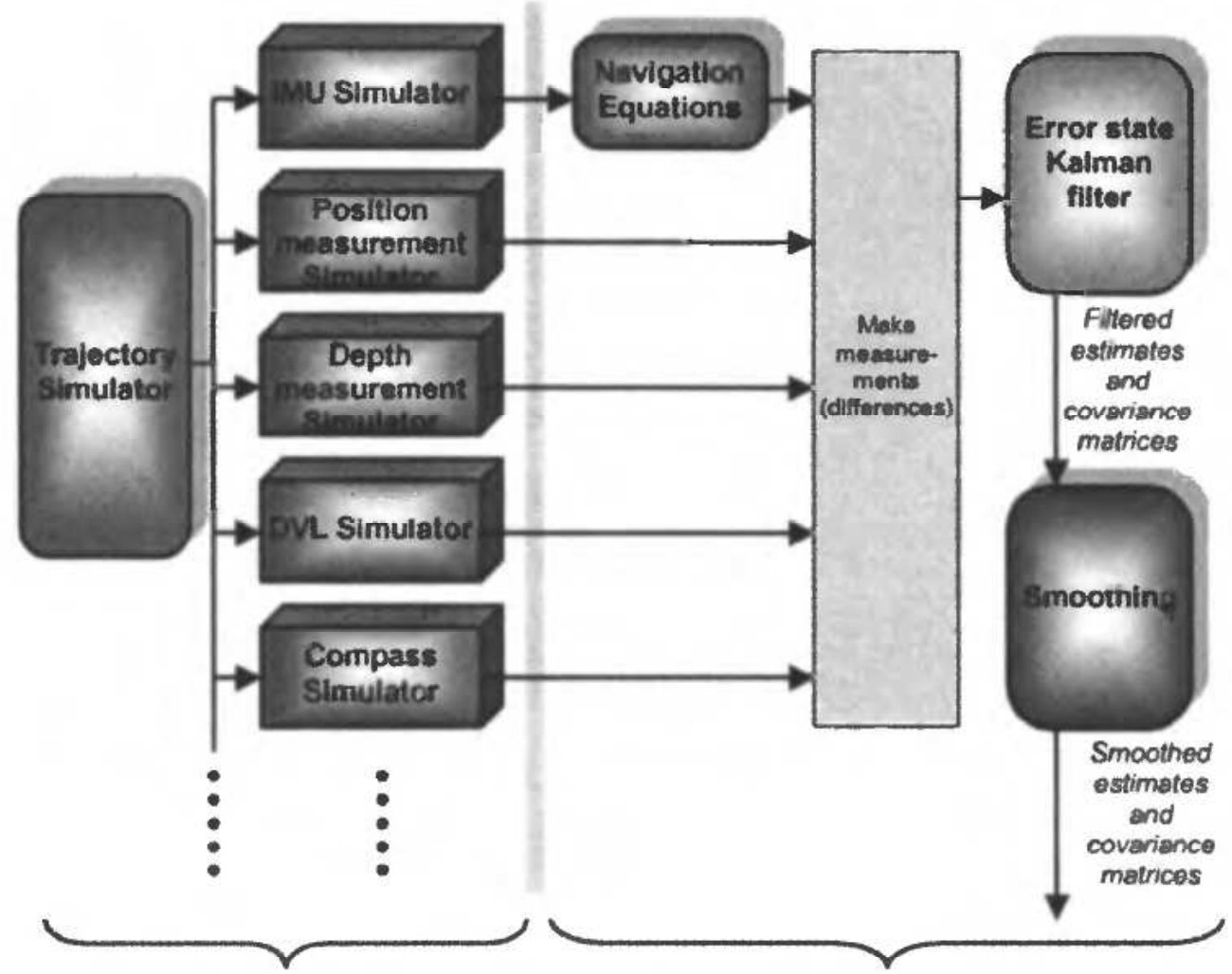

Simulator (can be replaced by real measurements)

\section{Estimator (can interface simulated or real measurements)}

Figure 3. NavLab structure.

- Navigation system research and development

- Navigation system accuracy analysis

- Navigation data post-processing

NavLab consists of a Simulator and an Estimator, see Figure 3. The Simulator can simulate any vehicle trajectory and a selected set of sensor measurements. The Estimator will, based on the available measurements, produce filtered and smoothed optimal estimates of position, velocity, attitude and sensor errors.

Prior to implementation in the HUGIN real-time navigation system, NavLab is used for algorithm research and development, simulation and testing. NavLab is also used for navigation system accuracy analysis and mission planning (even by HUGIN customers).

Since the Estimator works equally well on simulated and real measurements, NavLab is well suited and extensively used to produce optimal post-processed navigation results from HUGIN missions. When time and cost constraints allow, post-processed results are preferred to the real-time estimation results, since both the estimation accuracy and the integrity are improved. The increased accuracy is due to the use of smoothing, which is an optimal estimation technique that utilizes both past and future measurements. Smoothing is especially effective when position 

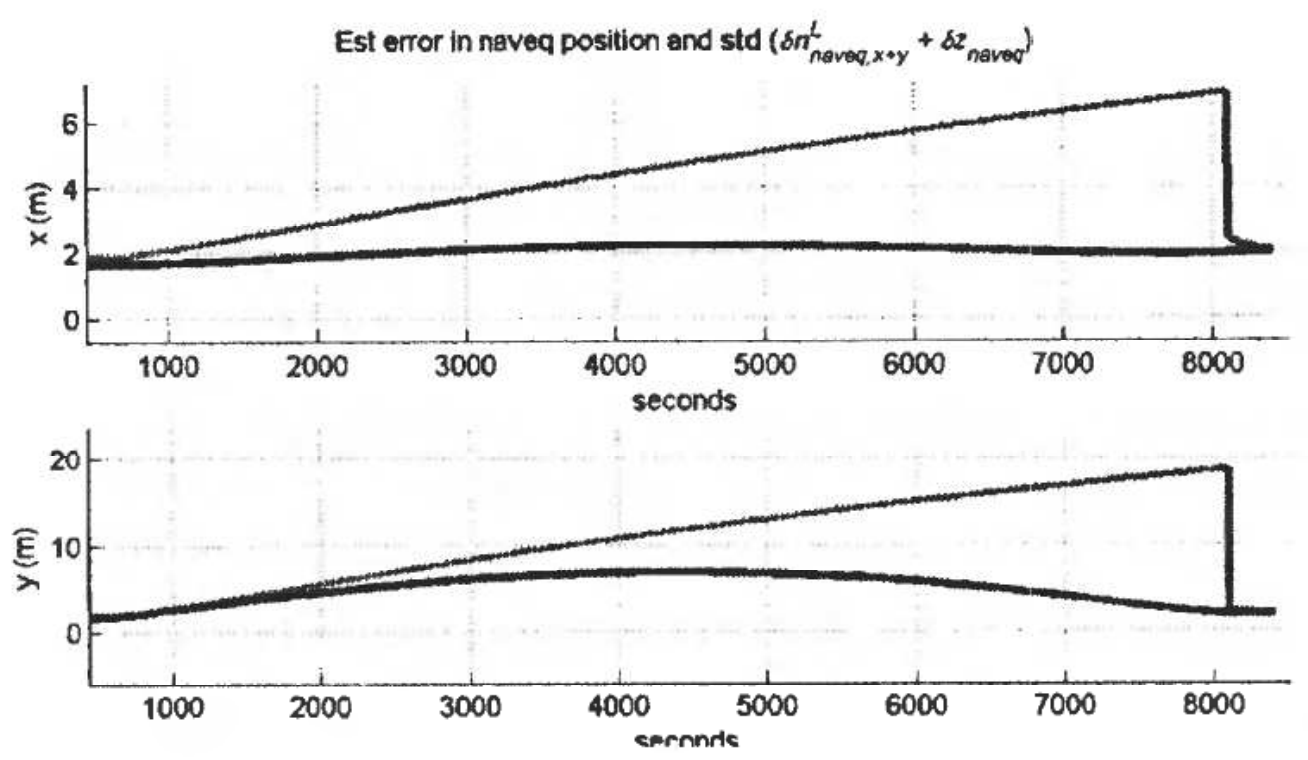

Figure 4. The effect of navigation post-processing when running a straight trajectory with GPS fix every $15 \mathrm{~km}$. Top graphs: real-time position accuracy $(1 \sigma)$. Bottom graphs: postprocessed position accuracy $(1 \sigma) . x$ and $y$ in local level (L) corresponds roughly to North and East direction.

updates are scarce, which is the case with GPS surface fixes, terrain navigation with few reference areas and scattered underwater transponders. In Figure 4 the effect of navigation post-processing when running a $15 \mathrm{~km}$ straight-line trajectory with GPS fix at the end is shown. The effect is less, but still substantial when running a lawn mower pattern (Jalving et al., 2003a).

The HUGIN real-time integrated inertial navigation system comes with extensive systems for integrity check. This is of crucial importance to safeguard against jamming, multipath effects, internal sensor failures etc. However, if the integrity mechanisms should fail to detect a navigation sensor wild point or degraded sensor performance, the real-time navigation estimates can be seriously affected. An important feature of navigation post-processing is increased navigational integrity and increased ability to recover faulty data sets. The smoothing algorithm is in general more robust against degraded sensor performance than the real-time Kalman filter and degraded sensor data sets can be filtered and improved.

NavLab has been extensively used by numerous international research groups and commercial mapping companies since 1999.

\section{Use of Navigation Toolbox in Different Applications}

An AUV operator will tailor the use of the integrated inertial navigation system to his specific needs and requirements. However, to illustrate the versatility of the HUGIN toolbox of navigation techniques, Table 4 suggests typical use of the navigation system in different applications.

GPS surface fix is the obvious, easy and accurate method for position update when water depth and covertness requirements allow. In deep water the actual AUV traveling time makes GPS fixes undesirable. Furthermore, loss of DVL bottom track will reduce the effect of the position fix due to the INS drift when diving. 
Table 4. Typical use of the HUGIN navigation system in different AUV applications.

\begin{tabular}{ll}
\hline Application & Navigation System Use \\
\hline Detailed seabed mapping & DVL aided INS \\
& DGPS-USBL position aiding \\
& NavLab post-processing \\
Environmental monitoring and research & DVL aided INS \\
& GPS surface fix \\
Inspection work for offshore industry & DVL aided INS \\
& UTP \\
MCM home areas-shalluw water & DVL aided INS \\
& GPS surface fix \\
MCM home areas-deep water & DVL aided INS \\
& UTP \\
REA low visibility & Terrain navigation \\
& DVL aided INS \\
REA-covert & GPS surface fix \\
& NavLab post-processing \\
& DVL aided INS \\
MCM denied areas (REA) & UTP CDN \\
\end{tabular}

Not mentioned in Table 4, UTP in concert with DGPS-USBL can potentially increase position accuracy for detailed seabed mapping in deep waters.

For MCM in home areas, accurate digital terrain models will be available for terrain navigation. In home areas, underwater transponders can also be pre-deployed in strategic locations.

Navigation strategies for REA operations are thoroughly analyzed in Jalving (2003a). Covert REA operations typically involves advanced concepts such as UTP CDN (Section 2.7.2) and CMN (Section 2.8.3).

\section{Operational Results}

\subsection{Detailed Seabed Mapping for Offshore Industry}

In detailed seabed mapping for the offshore industry DGPS-USBL position updates is the preferred method to obtain maximum position accuracy. In Jalving et al. (2003b) position accuracies in the final digital terrain models in water depths down to $3000 \mathrm{~m}$ have been thoroughly analyzed.

IIUGIN 3000 position accuracy was verified in comnercial mapping operations in varying water depths in the Gulf of Mexico in March 2001. The method used was mapping a known object, typically a wellhead, multiple times with reciprocal lines in different directions ('wagon wheel' pattern) and observe the position variance of the wellhead observations in the final DTMs. Applying NavLab post-processing a position accuracy of $2 \mathrm{~m}(1 \sigma)$ in $1300 \mathrm{~m}$ water depth and $4 \mathrm{~m}(1 \sigma)$ in $2100 \mathrm{~m}$ water depth was demonstrated. See Figure 5 for results in $1300 \mathrm{~m}$ water depth. 


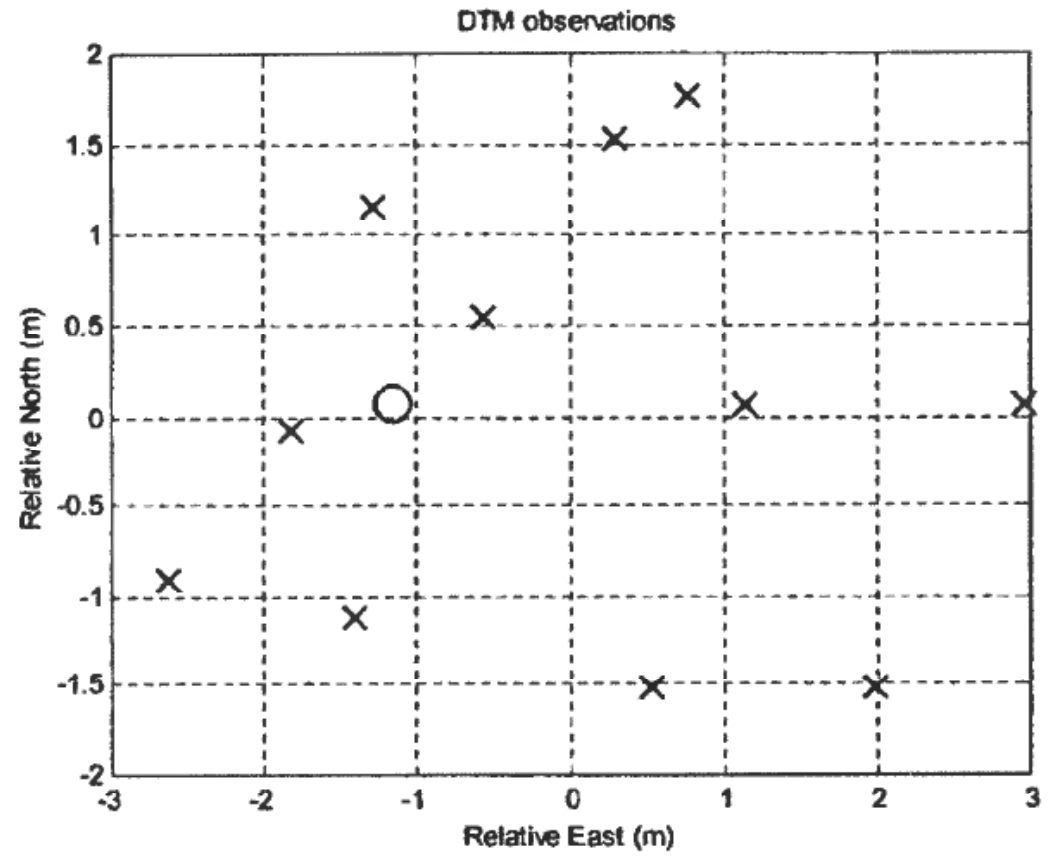

Figure 5. HUGIN 3000 position accuracy results. Crosses: Position estimates of different DTM wellhead observations in $1300 \mathrm{~m}$ water depth. Standard deviation in North is $1.2 \mathrm{~m}$, in East $1.7 \mathrm{~m}$.

\subsection{REA Mission with Norwegian Navy}

In August 2002, the Royal Norwegian Navy completed upgrade of a permanent HUGIN infrastructure on its KNM Karmøy mine countermeasures vessel. KNM Karmøy and HUGIN I are regularly used in operations of actual military worth (Hagen et al., 2003a). In Figure 6, the HUGIN I trajectory from a mission with KNM Karmøy in May 2003 is shown. HUGIN I was running an autonomous REA type of mission navigating with DVL aided INS and GPS surface fixes at regular intervals. HUGIN I was equipped with a $1 \mathrm{nmi} / \mathrm{h}$ type IMU (Table 1), a $300 \mathrm{kHz}$ DVL (Table 2) and a GPS SPS receiver. In Figure 7 difference between GPS and HUGIN INS is shown. When getting position fixes, the HUGIN INS position converges towards the GPS position. Considering the accuracy of a $300 \mathrm{kHz}$ DVL and GPS SPS (not differential), the results are very good. Navigation accuracy inbetween the GPS fixes can be further improved with NavLab post-processing, as explained in Section 2.9.

\subsection{Underwater Transponder Positioning}

A number of UTP sea trials were performed outside Horten. Norway, March 2003, with very good results. In Figure 8 the HUGIN trajectory and a picture of the deployed underwater transponder is shown. The relatively large size of the transponder is mainly due to a large battery pack and buoyancy material needed for retrieving the transponder.

HUGIN navigated in $180 \mathrm{~m}$ water depth with UTP as the only source for position updates. Post-mission, the navigation data was compared to independent DGPS- 


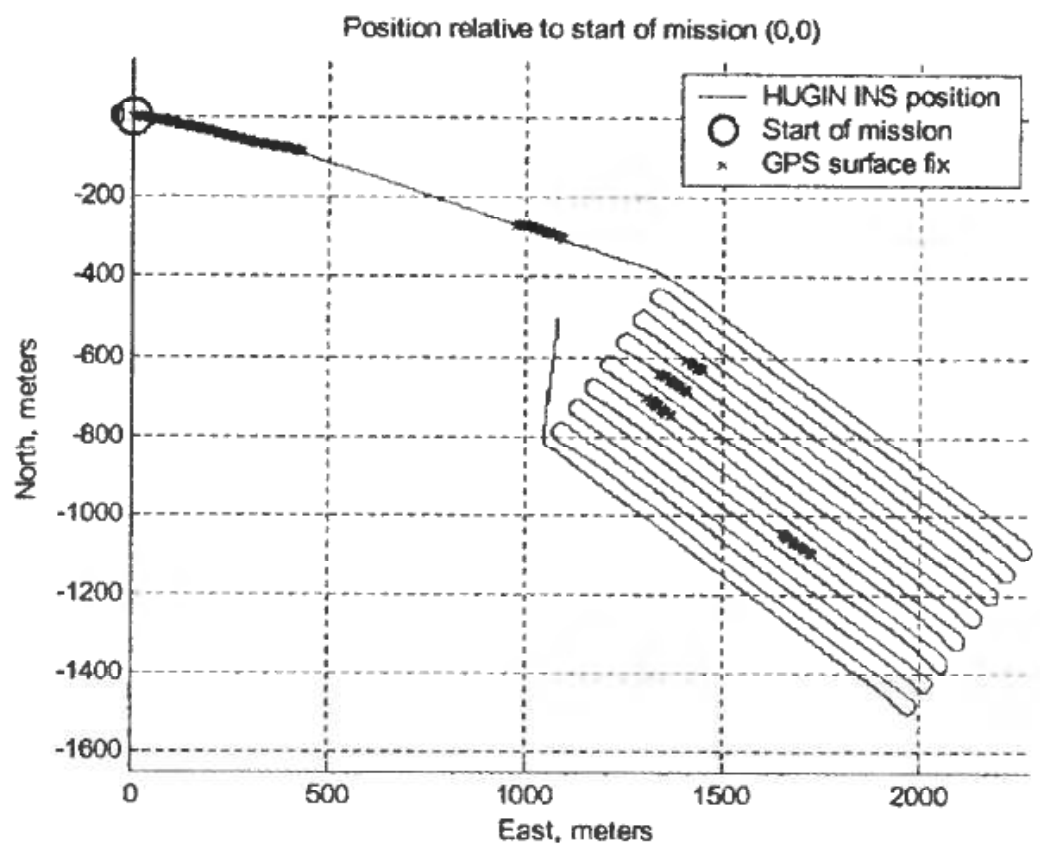

Figure 6. HUGIN I trajectory in autonomous mission from KNM Karmøy May 2003.
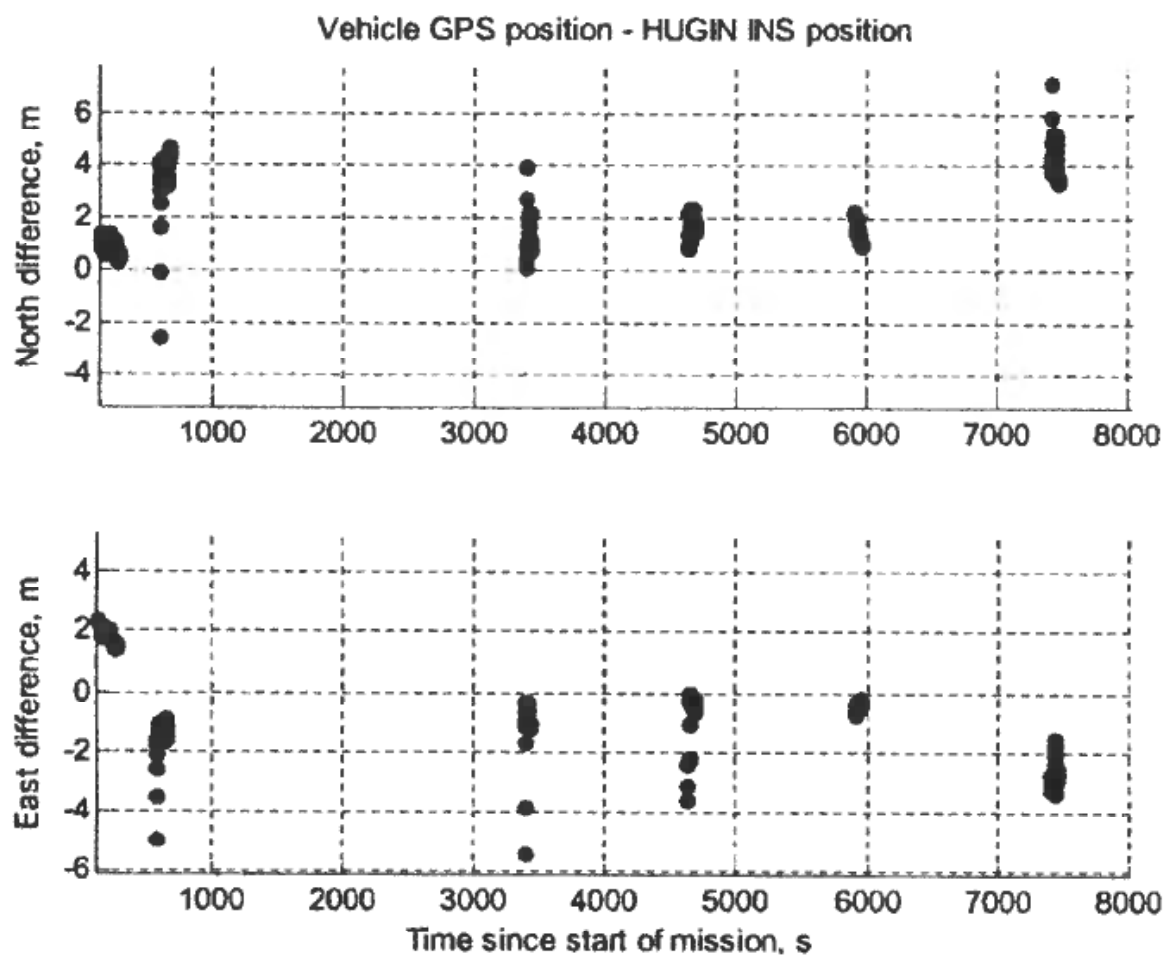

Figure 7. Difference between vehicle GPS and HUGIN integrated navigation system position estimate. When getting position fixes, the HUGIN INS position, converges towards the GPS position. 

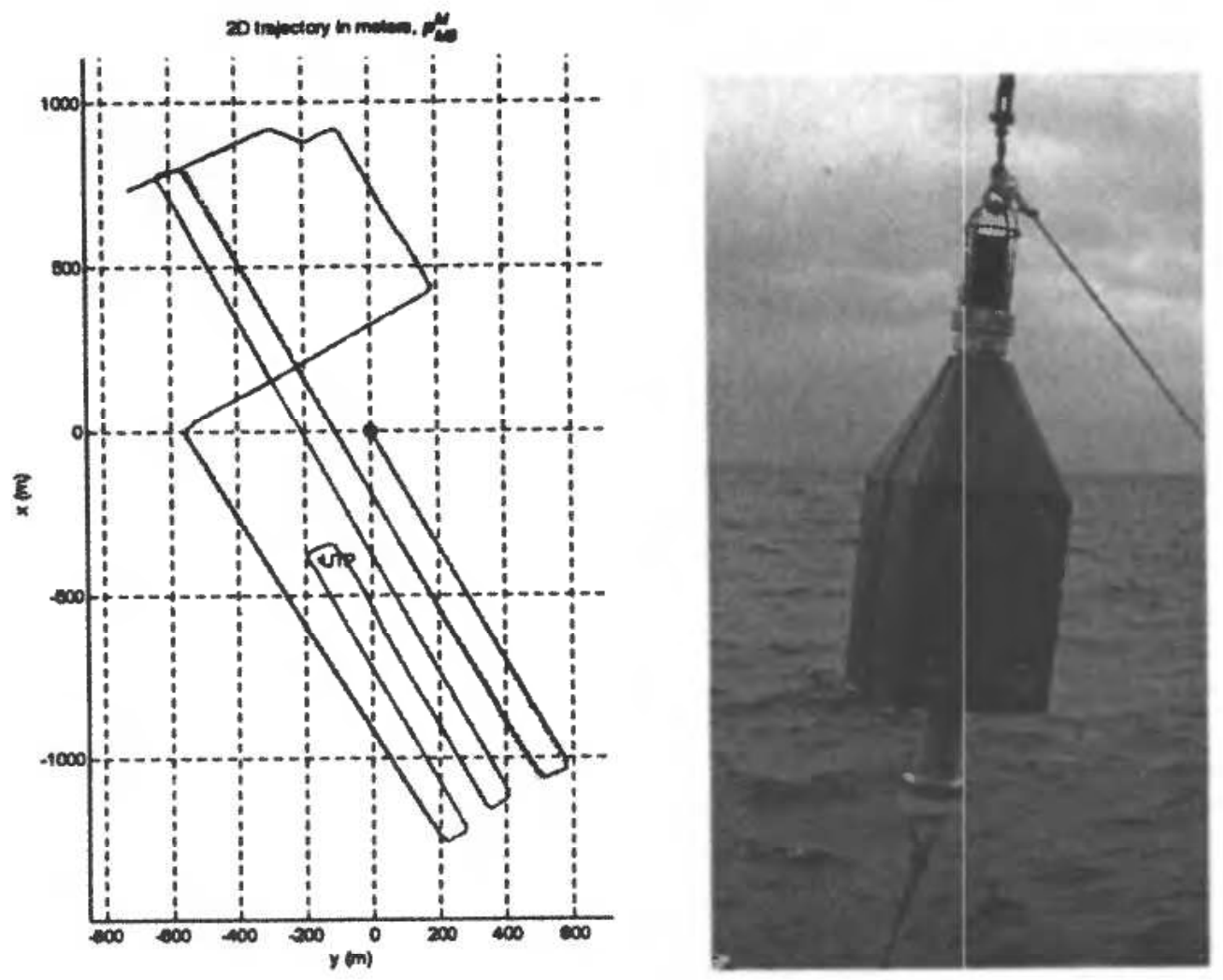

Figure 8. Left: HUGIN 2D trajectory in UTP sea trial. UTP was deployed at $x=-396 \mathrm{~m}$, $y=151 \mathrm{~m}$ (relative coordinates). Right: Underwater transponder used in sea trial.

USBL data stored on the survey vessel. The average difference between the two data sets in North and East was $2.2 \mathrm{~m}$ and $2.6 \mathrm{~m}(1 \sigma$, RMS). When NavLab postprocessing (smoothing) was applied, the difference reduced to $1.2 \mathrm{~m}$ in North and $1.5 \mathrm{~m}$ in East (1 $\sigma$, RMS). This is very close to the accuracy of the DGPS-USBL system. Figure 9 shows the difference between DGPS-USBL position estimate and the UTP post-processed navigation solution.

\subsection{Terrain Navigation}

The HUGIN terrain correlation system described in Section 2.8.1, is currently tested on recorded data from HUGIN missions conducted in a test area outside Horten in the Oslo fjord. The test area was surveyed by FFI's research vessel HU Sverdrup II in January 2001. A high quality DTM of $10 \mathrm{~m}$ resolution was produced. This DTM is statistically independent of the bathymetric data collected by HUGIN I, which is very important with respect to realistic testing of terrain navigation algorithms.

A data player plays the recorded real-time navigation solution and MBE and DVL bathymetric data. Except for the data player, the system is identical to the realtime version, which is due for the first sea trials in August 2003.

Figure 10 shows the contour lines of the inverse of the resulting correlation surface of the TERCOM algorithm for a position fix. Each fix is rated by a confidence 

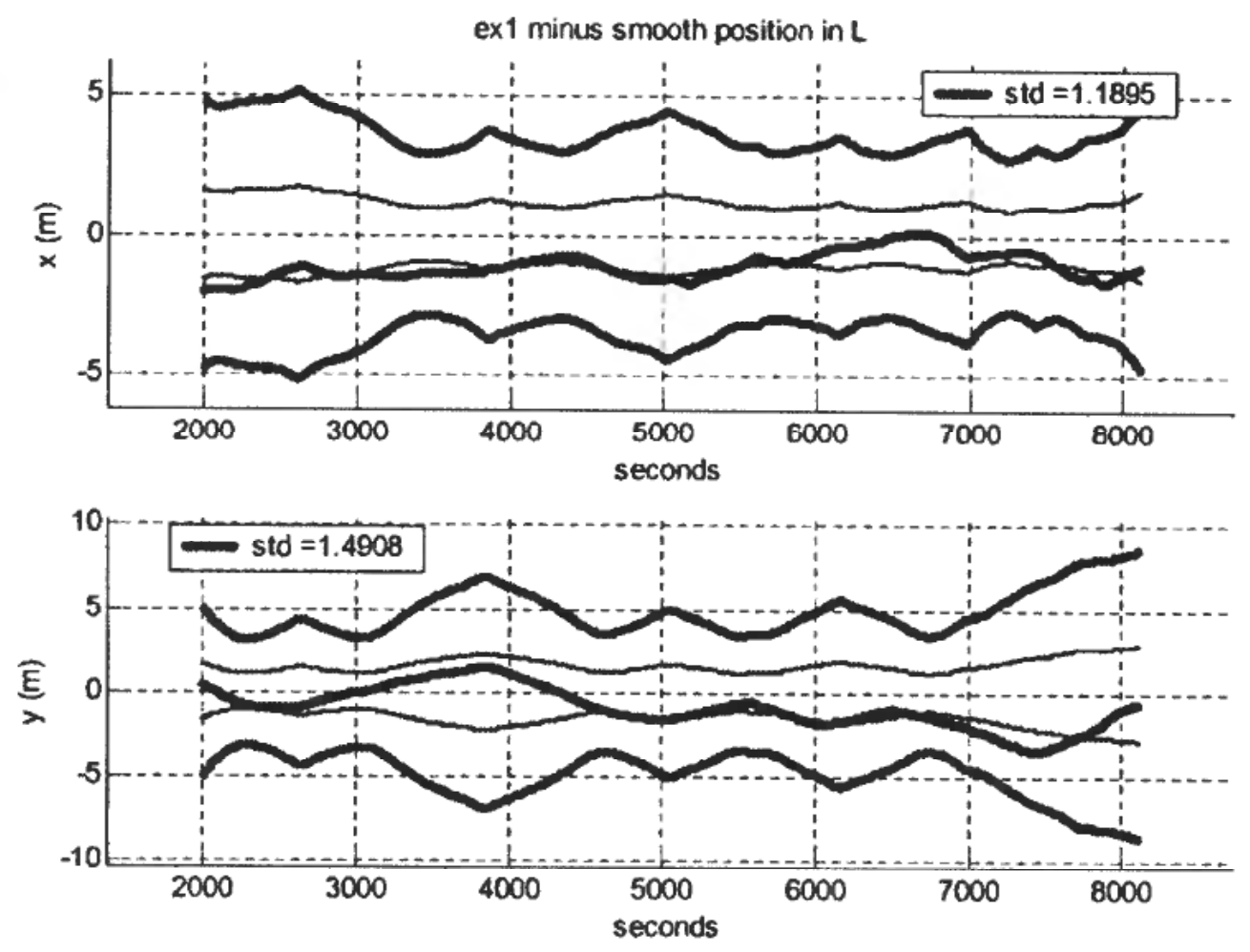

Figure 9. Results from UTP sea trial. Middle graph: difference between UTP post-processed navigation solution and independent DGPS-USBL position estimates. Surrounding graphs are $1 \sigma$ and $3 \sigma$ estimated uncertainty of the difference.

value 0 (low) to 1 (high). This value indicates stability of the fix and the presence of possible multiple solutions. For each fix an estimate of position standard deviation in northern and southern direction, along with the position covariance, are calculated using the correlation surface.

\section{Summary}

The main purpose of this paper has been to present the HUGIN integrated inertial navigation system and the extensive toolbox of navigation techniques, which has been designed to meet the navigation requirements for a broad variety of civilian and military AUV applications.

The core navigation system consists of a low drift velocity aided inertial navigation system based on a $1 \mathrm{nmi} / \mathrm{h}$ class IMU and an accurate DVL. There are several ways to counter the position error growth of a DVL aided INS. Cancelling of error growth with a lawn mower pattern is a very useful technique (Section 2.2.3).

If development work succeeds, a velocity measurement based on SAS technology can provide a leap in performance of velocity aided inertial navigation, allowing longer time intervals between position updates.

GPS surface fixes is the obvious, easy and accurate method for position updates when moderate water depths and covertness requirements allow.

For detailed seabed mapping operations in deep water, DGPS-USBL is the 


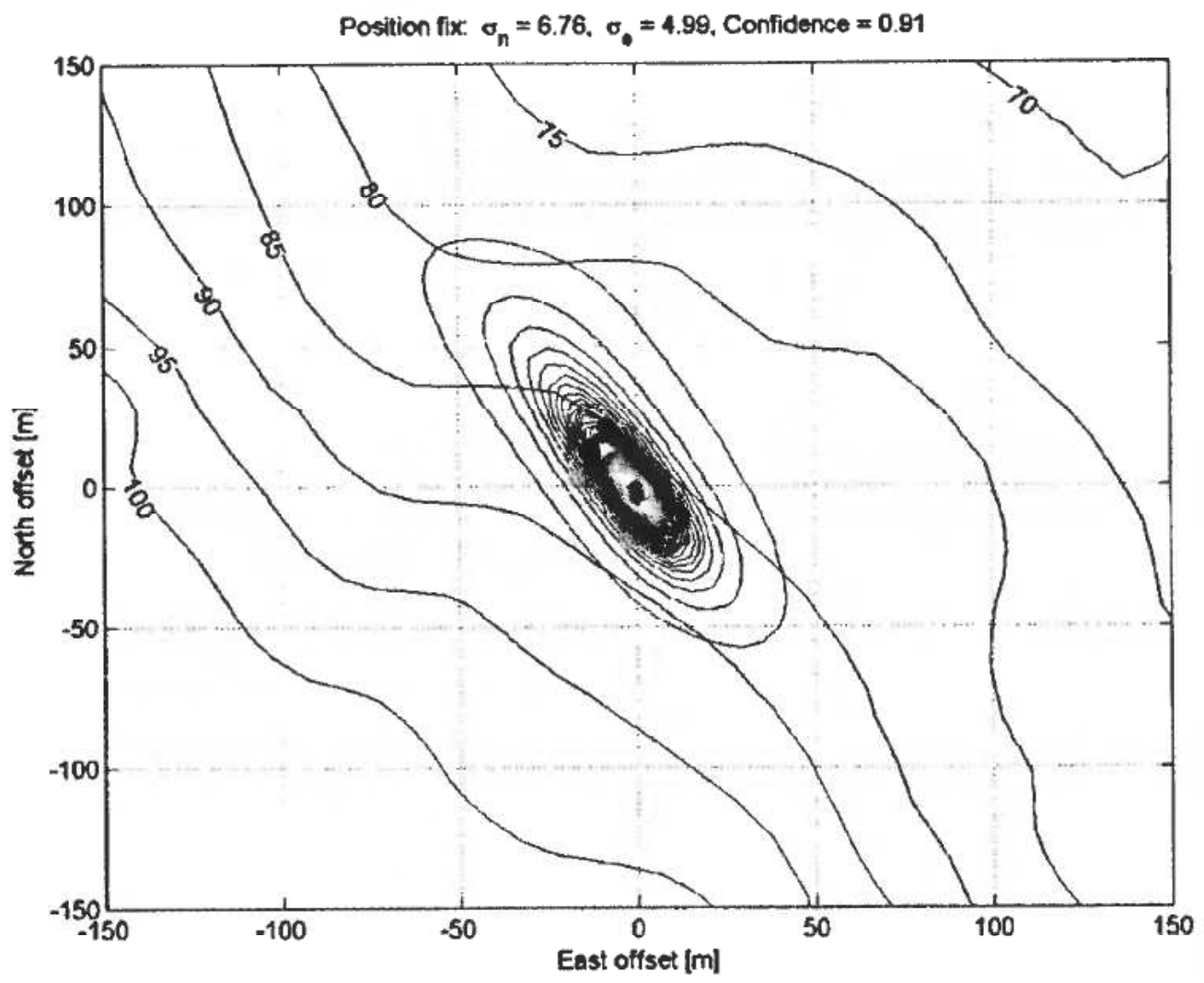

Figure 10. The correlation surface contour lines overlaid the DTM contour lines for a $300 \mathrm{~m} \times 300 \mathrm{~m}$ area. HUGIN I's position estimate (considered true position) is in the origin of this grid. Notice that the uncertainty of the fix is greater in the direction along the contour lines than across, indicating the importance of the position fix covariance.

preferred method for obtaining maximum position accuracy. HUGIN 3000 demonstrated in March $20012 \mathrm{~m}(1 \sigma)$ position accuracy in $1300 \mathrm{~m}$ water depth and $4 \mathrm{~m}$ $(1 \sigma)$ position accuracy in $2100 \mathrm{~m}$ water depth (with NavLab post-processing). To our knowledge, this accuracy has not yet been matched by any other survey AUV.

Underwater transponder positioning and terrain navigation allow for submerged position updates in autonomous missions. With only one transponder necessary for operation, UTP provides larger operational area and reduced deployment cost compared to LBL. UTP has in sea trials demonstrated very good accuracy (Section 4.3). Next development step is to facilitate concurrent deployment and navigation (UTP CDN, see Section 2.7.2).

Bathymetric terrain navigation is an appealing method for submerged position updates since bathymetric data from a standard AUV sensor suite is utilized: DVL, MBE, altimeter or interferometric sonar. In many scenarios, a digital terrain model will be available and actually used in mission planning. Next development step involves techniques for concurrent mapping and navigation (CMN).

Navigation post-processing maximizes the position accuracy and provides increased integrity check to a collected data set, features of crucial importance for deepwater detailed seabed mapping. Post-processing is especially effective when 


\begin{tabular}{|l|}
\hline \multicolumn{1}{|c|}{ HUGIN Navigation Toolbox } \\
\hline $\begin{array}{l}\text { DVL aided INS } \\
\text { - } \\
\text { Mission pattern for cancelling of error growth } \\
\text { Estimation and compensation of DVL error }\end{array}$ \\
\hline SAS velocity aiding \\
\hline GPS surface fix \\
\hline DGPS-USBL \\
\hline $\begin{array}{l}\text { Underwater transponder positioning (UTP) } \\
\text { - }\end{array}$ Navigation with pre-deployed transponders \\
Concurrent deployment and navigation (CDN) \\
\hline $\begin{array}{l}\text { Terrain navigation } \\
\text { Navigation with known DTM } \\
\text { Concurrent mapping and navigation (CMN) }\end{array}$ \\
\hline $\begin{array}{l}\text { NavLab } \\
\text { Navigation post-processing } \\
\text { analysis }\end{array}$ \\
\hline
\end{tabular}

position fixes are scarce, making it very attractive for covert REA applications. The NavLab Simulator can be used for navigation system accuracy analysis and can thus been an important tool in mission planning.

With the exception of SAS velocity aiding, UTP CDN and CMN, all the navigation techniques described in this paper is working commercially available technology (bathymetric terrain navigation is being tested in sea trials at time of writing). Furthermore, the HUGIN navigation system has in real applications onboard civilian survey vessels and on a navy mine countermeasures vessel demonstrated very good performance.

\section{References}

Bergman, N. Jung, L. \& Gustafsson, F. (1999). Terrain navigation using Bayesian statistics, IEEE Control Systems Magazine, 19(3), pp. $33-40$.

Chance, T. C., Kleiner, A. A. \& Northcutt, J. G. (2000). The HUGIN 3000 AUV, Sea Technology, 41(12), pp. 10-14.

GADE, K. (2003). NavLab User Guide, FFI/Report 2003/02128, Norwegian Defence Research Establishment.

GADE, K. (2004/5). NavLab, a Generic Simulation and Post-processing Tool for Navigation, paper to be published in 2004/2005.

GOLDEN, J. P. (1980). Terrain contour matching (TERCOM): a cruise missile guidance aid, Image Processing for Missile Guidance, 238, pp. 10-18.

HAGen, O. K. \& HaGeN, P. E. (2000). Terrain referenced integrated navigation systems for underwater vehicles, SACLANTCEN conference proceedings CP-46, NATO SACLANT Undersea Research Centre. 
Hagen, P. E., Størkersen, N., Vestgård, K., Kartvedt, P. \& Sten, G. (2003a). Operational military use of the HUGIN AUV in Norway, Proceedings from UDT Europe 2003, Malmö, Sweden.

Hagen, P. E., Størkersen, N., Vestgård, K. \& Kartvedt, P. (2003b). The HUGIN 1000 Autonomous Underwater Vehicle for Military Applications, Proceedings from Oceans 2003, San Diego, CA, USA.

JALVING, B., Bovio, E. \& GADE, K. (2003a). Integrated inertial navigation systems for AUVs for REA applications, SACLANTCEN conference proceedings from MREP 2003, NATO SACLANT Undersea Research Centre

Jalving, B., Vestgård, K. \& Størkersen, N. (2003b). Detailed seabed surveys with AUVs, in Technology and Applications of Autonomous Underwater Vehicles. Edited by Gwyn Griffiths. Taylor \& Francis, London and New York, pp. 179-201.

RD INSTRUMENTS (2003). Workhorse Navigator Doppler Velocity Log (DVL), http:// www.dvlnav.com/pdfs/navbro.pdf

Vestgård, K., Hansen, R., Jalving, B. \& Pedersen, O. A. (2001). The HUGIN 3000 Survey AUV, ISOPE-2001, Stavanger, Norway. 